\title{
Complete Genome Sequence of a Highly Pathogenic Porcine Reproductive and Respiratory Syndrome Virus Variant
}

\author{
Chengbao Wang, Qin Zhao, Chao Liang, Lu Dang, Yuping Ma, Jiming Gao, Qiongyi Li, Baicheng Huang, Ning Kong, Chong Zhang, \\ and En-Min Zhou \\ Veterinary Immunology Research Institute of Northwest A \& F University, College of Veterinary Medicine, Northwest A \& F University, Yangling, Shaanxi, China
}

Following the 2006 outbreaks of the highly pathogenic porcine reproductive and respiratory syndrome, the causative agent was identified as the highly pathogenic porcine reproductive and respiratory syndrome virus (HP-PRRSV). To investigate whether the HP-PRRSV variant continues circulating and accelerating evolution, we sequenced and analyzed the complete genome of the identified HP-PRRSV field strain SD16. The sequence data indicate that the HP-PRRSV variant continues to prevail and accelerate evolution, especially in the nonstructural protein.

Pising orcine reproductive and respiratory syndrome virus (PRRSV) is a member of a group of enveloped RNA viruses belonging to the genus Arterivirus of the family Arteriviridae in the order Nidovirales $(2,3,4,5)$. Since its appearance in 2006 , highly pathogenic porcine reproductive and respiratory syndrome virus (HPPRRSV) has a characteristic of a discontinuous 30-amino-acid deletion in nonstructural protein 2 (NSP2) (8). In this study, a field strain of PRRSV was isolated and identifies as a HP-PRRSV variant named SD16 according to the protocol described previously (6). The complete genome of SD16 was sequenced and analyzed to further understand the molecular evolution of HPPRRSV.

The $5^{\prime}$ and $3^{\prime}$ ends of the SD16 genome were confirmed by the SMART RACE cDNA amplification kit (Clontech, Japan), and the other parts were generated by seven overlapping cDNA fragments. All sequencing was done with an ABI Prism 3730 sequencer (Applied Biosystems) and assembled using SeqMan software (DNASTAR Inc.). The complete genome of SD16 is 15,320 nucleotides (nt) in length [excluding the poly(A) tail] including the numbers of nucleotides for the following genes: 189 for the 5 ' untranslated region (UTR), 11,792 for the Rep gene, 771 for the GP2a gene, 222 for the GP2b gene, 765 for the GP3 gene, 537 for the GP4 gene, 603 for the GP5 gene, 525 for the M gene, 372 for the $\mathrm{N}$ gene, and 150 for the $3^{\prime}$ UTR. There are $87 \mathrm{nt}$ deletions in the most highly variable region of NSP2. Furthermore, we found $10 \mathrm{nt}$ mutations in the SD16 genome compared with the other PRRSV isolates deposited in GenBank, resulting in 3 unique amino acid changes in the Rep gene.

The 5' and 3' UTRs of PRRSV carry signals which ensure the recognition and efficient translation of the genomic RNA by the cellular translation apparatus as well as its replication by the viral RNA-synthesizing complex $(1,7)$. The $5^{\prime}$ UTR includes the leader transcription regulatory sequence (TRS) which is important for generation of subgenomic mRNA (7). The UTRs are highly conserved in PRRSV while $1 \mathrm{nt}$ is mutated in the $3^{\prime}$ UTR of the SD16 genome, which could be used as the markers to differentiate SD16 from the other PRRSV isolates. Moreover, there are six-body TRSs spreading through the SD16 genome located at about 0.02 to 0.24 $\mathrm{kb}$ upstream from the start codons of open reading frames 2 to 7 (ORF2 to -7) and the conserved sequences in the SD16 genome are the hexameric motifs $5^{\prime}-(\mathrm{G} / \mathrm{U})(\mathrm{A} / \mathrm{G})(\mathrm{A} / \mathrm{G}) \mathrm{CC}-3^{\prime}$.

Our data and the previously reported data indicate that the
HP-PRRSV variant continues prevailing and accelerating evolution in China $(8,9,10)$. Results of the SD16 sequence will enhance not only our understanding of the PRRSV evolutionary mechanism but also that of arterivirus.

Nucleotide sequence accession number. The full genomic sequence of SD16 was deposited in GenBank under accession no. JX087437.

\section{ACKNOWLEDGMENTS}

This work was supported by grants from the National Natural Science Foundation of China (U0931003/L01, 30871857/C1803), the National Transgenic Project of China (2009ZX08009-147B), and the National High-Tech R\&D Program of China (2009AA10Z111).

\section{REFERENCES}

1. Beerens N, Snijder EJ. 2006. RNA signals in the $3^{\prime}$ terminus of the genome of Equine arteritis virus are required for viral RNA synthesis. J. Gen. Virol. 87:1977-1983.

2. Cavanagh D. 1997. Nidovirales: a new order comprising Coronaviridae and Arteriviridae. Arch. Virol. 142:629-633.

3. Keffaber KK. 1989. Reproductive failure of unknown etiology. Am. Assoc. Swine Pract. Newsl. 1:1-10.

4. Mayo MA. 2002. A summary of taxonomic changes recently approved by ICTV. Arch. Virol. 147:1655-1663.

5. Neumann EJ, et al. 2005. Assessment of the economic impact of porcine reproductive and respiratory syndrome on swine production in the United States. J. Am. Vet. Med. Assoc. 227:385-392.

6. Qiu HK, Cao BL, Xiao YH, Zhou EM. 2010. Isolation and sequence analysis of PRRSV SD-TA mutational strain in southwest China. J. Agric. Sci. 23:1698-1702.

7. Snijder EJ, Meulenberg JJ. 1998. The molecular biology of arteriviruses. J. Gen. Virol. 79:961-979.

8. Tong GZ, et al. 2007. Highly pathogenic porcine reproductive and respiratory syndrome, China. Emerg. Infect. Dis. 13:1434-1436.

9. Wu JJ, et al. 2012. Complete genome sequence of an overattenuated highly pathogenic porcine reproductive and respiratory syndrome virus. J. Virol. 86:6381-6382.

10. Zhou Z, et al. 2012. Complete genome sequence of two novel Chinese virulent porcine reproductive and respiratory syndrome virus variants. J. Virol. 86:6373-6374.

Received 22 May 2012 Accepted 4 June 2012

Address correspondence to En-Min Zhou, zhouem@nwsuaf.edu.cn

Copyright @ 2012, American Society for Microbiology. All Rights Reserved.

doi:10.1128/JVI.01281-12 